\title{
From chaos to stability in soliton mode-locked fiber laser system
}

\author{
M. A. Sharif ${ }^{a, *}$ and K. Ashabi ${ }^{b}$ \\ ${ }^{a}$ Urmia University of Technology, Faculty of Electrical engineering, \\ Optics and Laser engineering group, Band road, Urmia, Iran, 5716617165. \\ *e-mail: m.abdolahisharif@ee.uut.ac.ir \\ ${ }^{b}$ MEAPAC Service, L.L.C. One Research Court Suite 450, Rockville, MD 2850, United States.
}

Received 17 March 2021; accepted 16 April 2021

\begin{abstract}
Intracavity energy rate in a soliton mode-locked fibe laser is derived by solving the Haus master equation. The influenc of net gain, absorber response, saturation energy, nonlinearity and absorption are investigated on stable/unstable states. Intracavity modes include the zeroth, firs and higher order solitons. Accordingly, chaotic regime as well as breather modes is recognized as a conventional intracavity state. However, tuning the control parameters also results in a reverse bifurcation and thus returning to a stable state. Accordingly, a chaos-based encryption/decryption system is proposed taking the advantage of using a single-side control process; both the encryption and decryption procedures can be achieved by one of the actions of increasing/decreasing the control parameters.
\end{abstract}

Keywords: Soliton mode-locked fibe laser; chaos; stability; breather solution.

DOI: https://doi.org/10.31349/RevMexFis.67.061301

\section{Introduction}

Due to their outstanding features including the high gain efficien y, high power, low fabrication cost and compactness, soliton mode-locked fibe lasers have been known as essential tools for many applications like the pulse generation, optical communication, laser hardening, cutting and engraving [1-6]. Meanwhile, nonlinear dynamical stability analysis is an important point which has been contemplated through different studies. One crucial issue is the period doubling, a route to chaos which has been experimentally and theoretically demonstrated in soliton mode-locked fibe lasers [7-9].

In the nonlinear optical systems, the procedure of route to chaos begins with quasi-periodic state and then, proceeds within the form of an unstable state with exponential growing amplitude and unpredictable behaviour. Although chaos emerges as an instability in the laser output, it can be used as a basis for particular applications like the secure optical communication whereas the encryption procedure is based on interfering a chaotic map [10-12]. In spite of this, decryption stage needs the reverse chaotic map which can be hardly implemented.

In this study, the Haus-master equation is considered for deriving the intracavity energy rate in a soliton mode-locked fibe laser. The influence of absorber response, nonlinearity and gain are then investigated on the intracavity energy rate to determine the stable/unstable states. It is shown that the breather modes (higher order solitons) are most likely formed in consequence of the high gain/large nonlinearity and chaotic regime is thus, a conventional intracavity energy state. Finally, a procedure of reverse bifurcation is deduced and accordingly proposed for a convenient decryption procedure in chaos-based optical communication.

\section{Theory}

Soliton formation is considered as the main mechanism of pulse shaping in a soliton mode-locked fibe laser. Intracavity nonlinear dynamics can be described by the Haus master equation as given in Eq. (1) [13-17].

$$
\begin{aligned}
T_{R} \frac{\partial A(T, t)}{\partial T} & =-i D \frac{\partial^{2} A}{\partial t^{2}}+i \gamma|A|^{2} A \\
& +\left(g-l+D_{g} \frac{\partial^{2}}{\partial t^{2}}-q(T, t)\right) A(T, t),
\end{aligned}
$$

where $A(T, t)$ is the slowly varying pulse amplitude; $T$ is the time scaled to one cavity round trip $T_{R} ; t$ is the time scaled to pulse duration $\tau$. It should be noticed that $T$ and $t$ are independent and intrinsically irrelevant parameters. $D$ is the Group Velocity Dispersion (GVD) and $D_{g}$ is the gain dispersion; $\gamma$ is the nonlinear coefficien standing for the effect Self Phase Modulation (SPM); $g$ and $l$ are respectively the gain and loss indices respectively and $q(T, t)$ is the response of the absorber. For simplicity, $g-l$ is substituted by the quantity net gain $g_{N E T}$. Consecutively, the pulse energy can be written as Eq. (2).

$$
E=\int_{-T_{R} / 2}^{T_{R} / 2}|A(T, t)|^{2} d t .
$$

Moreover, energy rate equation is proved to be written as Eq. (3) [13-17].

$$
T_{R} \frac{\partial E}{\partial T}=2 g_{N E T}-2 q(E),
$$

in which

$$
g_{N E T}=\frac{g_{0, N E T}}{1+E / E_{s}}
$$


where $g_{0, N E T}$ is the primary net gain value and $E_{s}$ is neglected. is the saturation energy of the absorber. If the cavity possesses a slow saturable absorber, the minimum energy needed for the pulse formation can be obtained by Eq. (4) [13-17].

$$
E_{s}^{\min }=\sqrt[3]{\frac{q_{M} E_{s}}{\alpha_{T P A}}}
$$

where $q_{M}$ is the maximum absorber response and $\alpha_{T P A}$ is define to measures the two-photon absorption using Eq. (5) [13-17].

$$
\alpha_{T P A} \propto \frac{\alpha \gamma}{12 D}
$$

where $\alpha$ is the absorption due to the Rayleigh scattering. If the competition between GVD and SPM is balanced in Eq. (1), intracavity steady state will be the result. In this case, pulse duration is shown to be given as Eq. (6) [13-17].

$$
\tau=\frac{4|D|}{\gamma E}
$$

for which the pulse shape can then be obtained by Eq.(7).

$$
f(t)=\sqrt{\frac{1}{2 \tau}} \operatorname{sech}\left(\frac{t}{\tau}\right) .
$$

This solitary solution is resulted for the firs order solitons.

For a slow saturable absorber, the energy rate is obtained in terms of $T$ as given by Eq. (8) [13-17].

$$
\begin{aligned}
E(T) & =\frac{1}{2} \frac{g_{0, N E T}}{1+\frac{E\left(T-T_{R}\right)}{E_{s}}} \\
& \times\left(1-q_{M} \cos \left[\pi E\left\{T-T_{R}\right\}\right]\right) E\left(T-T_{R}\right) .
\end{aligned}
$$

The total absorber response is a combination of saturable and inverse saturable contributions as presented in Eq. (9)[13-17].

$$
q(T, t)=q_{s}(T, t)-q_{I}(T, t),
$$

where, the saturable part reads Eq. (10)[13-17].

$$
\frac{\partial q_{s}(T, t)}{\partial t}=-\frac{q_{s}-q_{s, M}}{t_{r}}-\frac{|A(T, t)|^{2}}{E_{s}} q_{s},
$$

where $q_{s, M}$ is the maximum response of saturable part and $t_{R}$ is the relaxation time of the absorber. One may consider a solution for Eq. (10) in the form of Eq. (11) if the slow saturable absorber is assumed.

$$
q_{s}=q_{s, M} \frac{1-\exp \left(-E / E_{s}\right)}{E / E_{s}} .
$$

On the other hand, the inverse saturable term can be written as Eq. (12)[13-17].

$$
q_{I}(t)=\alpha_{T P A} l_{e f f} \frac{|A(t)|^{2}}{a_{e f f}},
$$

where $l_{\text {eff }}$ and $a_{\text {eff }}$ is respectively the effective length and area of the absorber interacted with the laser beam.

If $q_{M}, \alpha, \gamma, g_{0, N E T}$ and subsequently $E_{s}$ remain small, a cw steady state will be deduced. Otherwise, soliton regime will be dominant if the saturation energy value $E_{s}$ becomes greater than the minimum energy $E_{s}^{\mathrm{min}}$ requiring the other mentioned parameters to be sufficientl large. A further increase in $E_{s}$ may lead to the violation of the balance between GVD and SPM. Consequently, soliton formation will then vanish. However, there can be a total average balance between the two factors within the long-time measurement while each of them alternatively surpasses in a period of the time. This results in a quasi-periodic state implying the formation of higher order solitons. If $E_{s}$ is more than a threshold value $\left(E_{t h}\right)$, chaotic regime will be deduced.

\section{Simulation results and discussion}

In order to perform a comparative representation, energy value $E$ and temporal parameters $T$ and $t$ are normalized in Eq. (9). Figure 1 shows the procedure of pulse generation within the cavity with respect to the intracavity energy state. Blue lines indicate the region for which the pulses can be formed. The white surface depicts the minimum energy above which the pulses can be formed, whereas the black surface illustrates the unstable region.

Meanwhile, we use the predictor-corrector split step Fourier method -which is a combination of explicit and im-

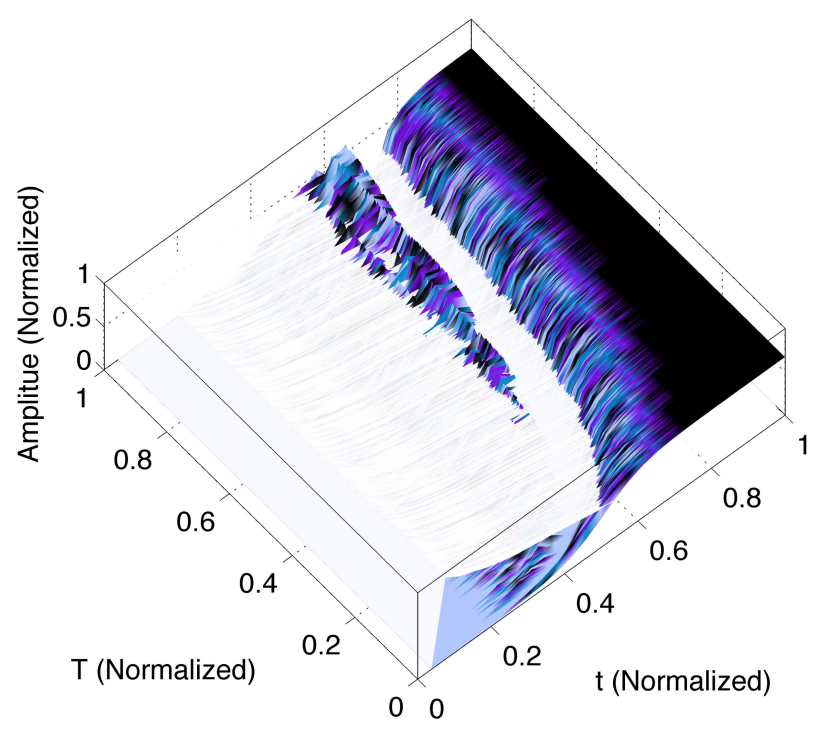

FIGURE 1. Pulse formation procedure with respect to intracavity energy states. 

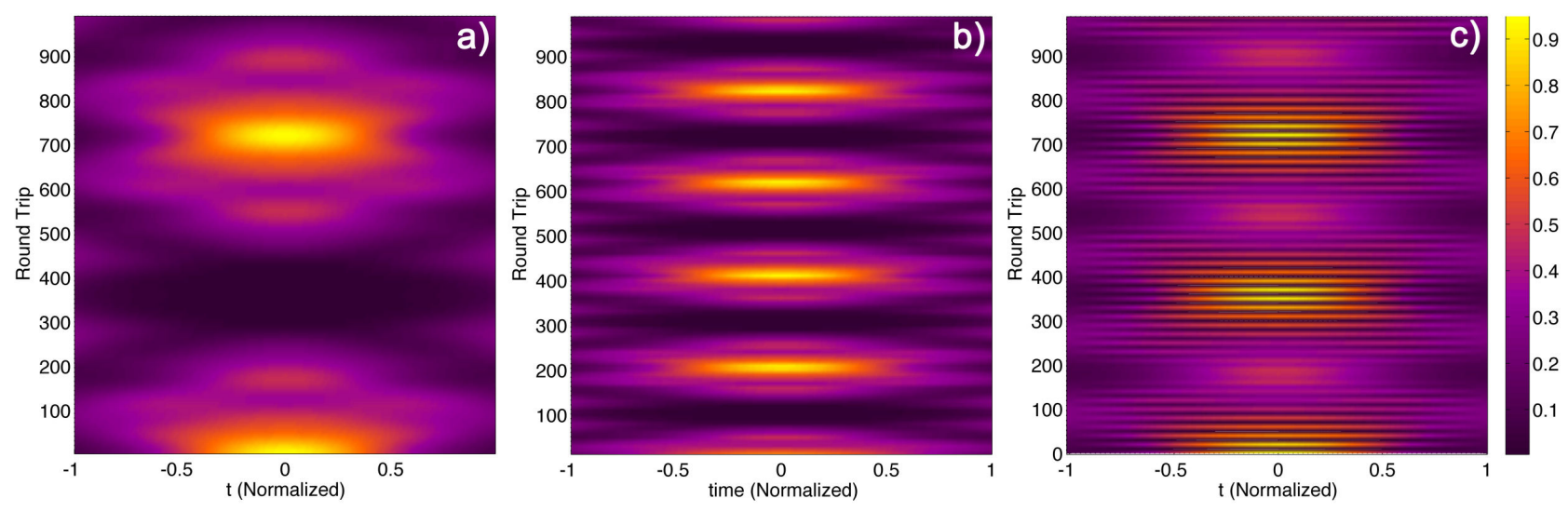

FIGURE 2. Intracavity illustration of a) zeroth order solitons, b) firs order solitons, c) breather modes.
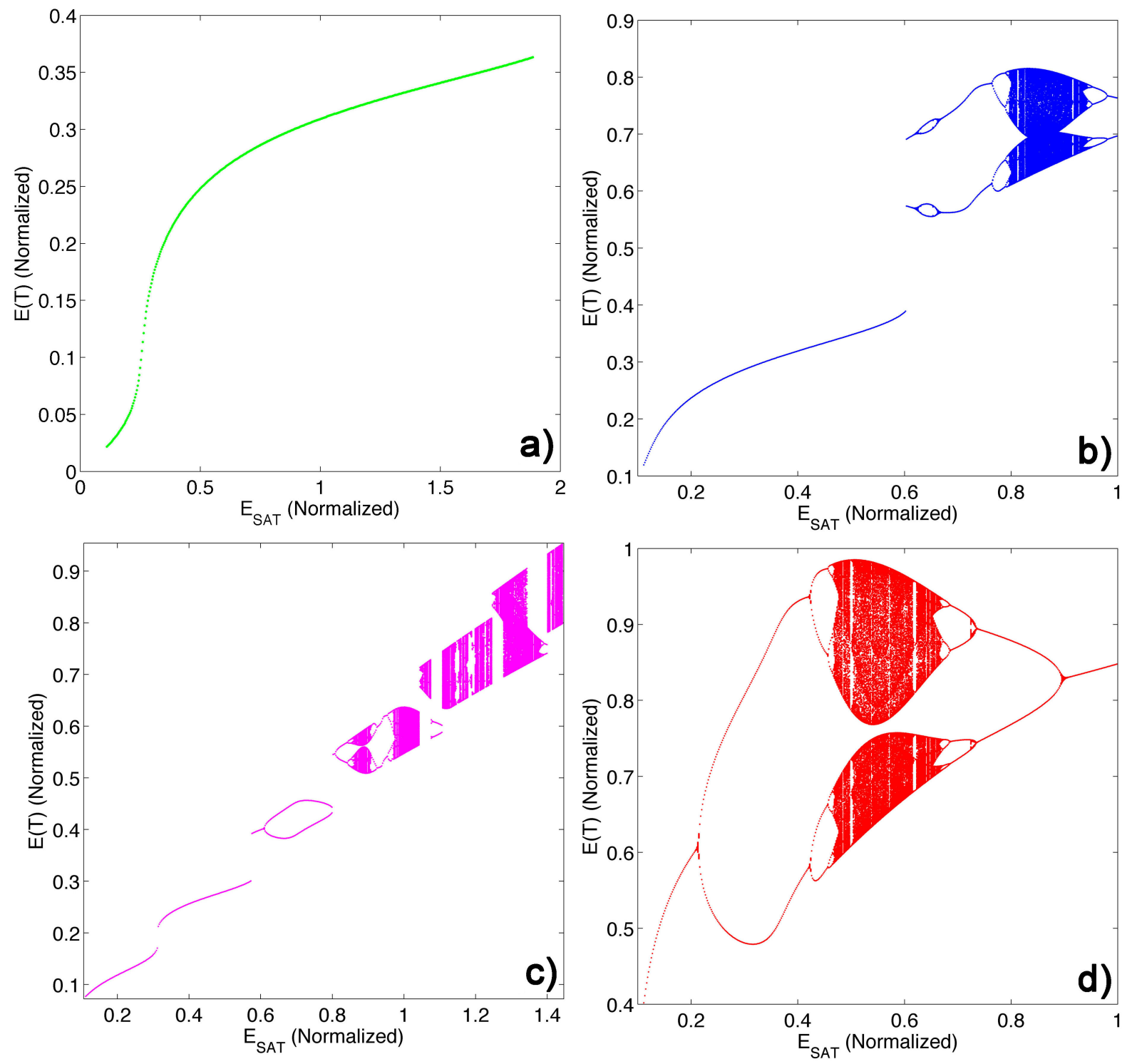

FIGURE 3. Bifurcation diagrams for a) $q_{M}=0.2, g_{0}=1.7$, b) $q_{M}=0.2, g_{0}=2.7$, c) $q_{M}=0.1, g_{0}=3.7$ d) $q_{M}=0.4, g_{0}=2.7$. 
plicit methods- to solve Eq. (1) numerically [18-19]. Figure 2 shows the results as the three pulse formation states categorized in accordance with the three energy zones presented in Fig. 2. For a slightly larger saturation energy than $E_{s}^{\mathrm{min}}$, zeroth order solitons appear (i.e. the solitons that are not yet suitable for the ultrafast pulse regime) (Fig. 2a)). If the saturation energy increases enough, the procedure of soliton formation will be faster and the $1^{\text {th }}$ order solitons will emerge (Fig. 2b)). Further increase in the saturation energy leads to the generation of higher order solitons (Fig. 2c)), a prior state to chaos.

In the next step, the influenc of the parameters $q_{M}$, and subsequently, $E_{s}$ is to be investigated on the route to chaos. Figure 3 shows the results as the bifurcation diagrams for different assumed compositions. For the lower values assumed parameters $\left(q_{M}=0.2, g_{0, N E T}=1.7\right)$, a cw stationary regime appears as anticipated. This includes the two lower and upper stable states as shown in Fig. 3a). An increase in
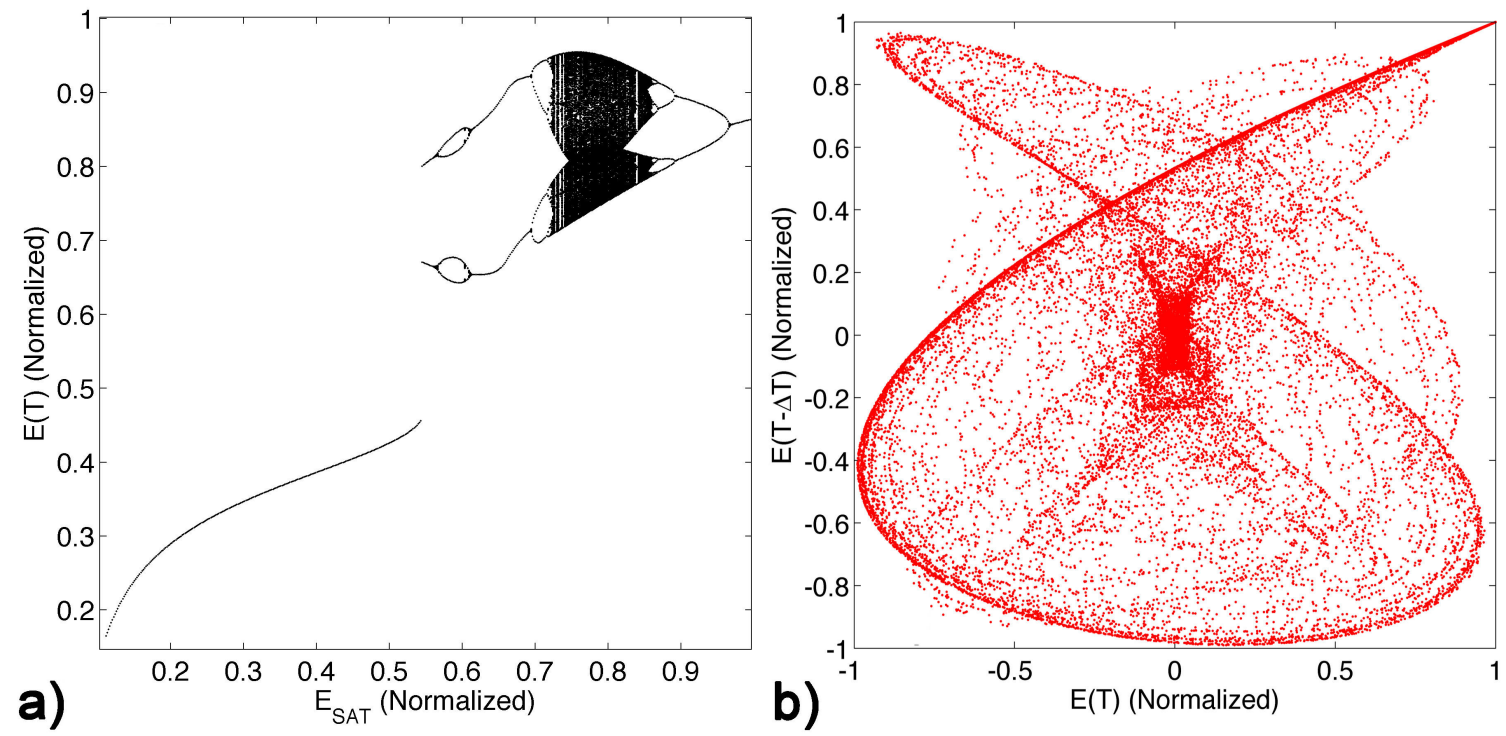

FIGURE 4. Bifurcation diagrams for a) $\gamma=3.2$ while other parameters are the same as assumed for Fig. 3a), b) its chaotic attractor.

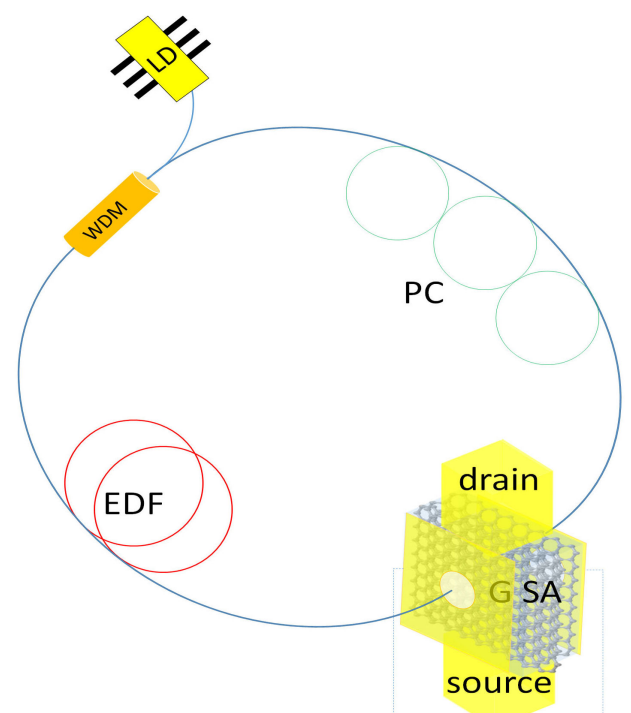

a)

Top, back gates

FIGURE 5. a) Schematic illustration of a graphene-assisted soliton mode-locked fibe laser system; EDF means Erbium-doped fiber WDM means the wavelength division multiplexing; PC indicates the polarization controller; LD indicates the pump laser diode; magnificatio depicts the FET structure based on multiple embedded graphene layers; $G$ SA denotes the graphene saturable absorber; b) Bifurcation diagrams obtained for variation of $\gamma$. 
$g_{0, N E T}\left(g_{0, N E T}=2.7\right)$ leads to the period doubling and then multiple periodic behaviour inferred as a route to the chaotic regime (Fig. 3b)). The situation will become more complex for the larger $g_{0, N E T}$ even if $q_{M}$ decreases (Fig. 3(c) obtained for $g_{0, N E T}=3.7$ and $\left.q_{m}=0.1\right)$. Moreover, if $g_{0, N E T}$ and $q_{M}$ further increase $\left(g_{0, N E T}=4.7\right.$ and $\left.q_{m}=0.2\right)$, a reverse bifurcation diagram will be resulted as shown in Fig. 3d). This is a conventional state in the cavity of the mode-locked fibe lasers and has been more or less shown theoretically and experimentally. Nevertheless, from Eqs. (11) and (12) one may deduce that the maximum absorber response can be written as Eq. (13) if the amplitude is normalized to 1.

$$
q_{M} \propto\left(1-\alpha_{T P A}\right) .
$$

Accordingly, Eq. (5) will suggest that the two control parameters $\alpha$ and $\gamma$ can be tuned to modify the system state. Figure $4 a$ ) shows the bifurcation diagram obtained for the larger nonlinear coefficien $\gamma=3.36$ in comparison to the modest nonlinear coefficien $\gamma=2$ assumed in Fig. 3a). Also, Fig. 3b) shows the corresponding chaotic attractor.

Indeed, the control parameters of $\gamma$ and $\alpha$ can be electrically or optically tuned by using a supplementary entity. For example, graphene-based mode-locked fibe lasers can provide such usefulness beside the other beneficia issues [2024]. Therefore, designing a both encryption and decryption system based on the chaos will be possible by adjusting the saturation energy $E_{s}$ or tuning the nonlinearity $\gamma$ or absorption $\alpha$. A schematic illustration of a graphene-assisted soliton mode-locked fibe laser system based on the previously proposed schemes are shown in Fig. 5a) whereas the magnificatio shows a FET structure using a multiple embedded graphene sheets to enhance the nonlinearity and absorption [25-26].

Variation of the $\gamma$ and $\alpha$ causes a modificatio in $E_{s}$ according to Eq. (4). Considering this fact, the bifurcation diagram obtained for $\gamma$ variations is shown in Fig. 5b).

Another important advantage is the single-side control process, i.e., there is no need to increase and decrease the nonlinear coefficient One action will be lonely sufficien to trigger both the encryption and decryption procedures. This will be crucially substantial if one notices that the reverse action cannot follow the same response in the nonlinear dynamical systems.

\section{Conclusion}

The Haus master equation has been solved for the cavity of soliton mode-locked fibe laser. It has been shown that the intracavity modes include the firs and higher order solitons. Accordingly, chaotic regime as well as breather modes have been recognized as the conventional state within the cavity. The process of controlling intracavity states is implemented by changing the characteristic parameters including the net gain, absorber response and also the control parameters of saturation energy, nonlinear and absorption coefficients While increasing the control parameters leads to the chaotic regime, it has been revealed that further increasing can result in a stable state inferred from the reverse bifurcation diagram. On this base, a chaos-based encryption/decryption system has been proposed. The system takes the advantage of using a single-side control process, i.e., the both encryption and decryption procedures can be achieved by increasing or decreasing the control parameters.
1. Y. Song, X. Shi, C. Wu, D. Tang and H. Zhang, Recent progress of study on optical solitons in fibe lasers, Appl. Phys. Rev. 6 (2019) 021313, https: //doi.org/10.1063/1. 5091811

2. M. N. Zervas, and A. Christophe, Codemard, High power fibe lasers: a review, IEEE J. Sel. Top. Quantum Electron. 20 (2014) 219, https://doi.org/10.1109/JSTQE. 2014.2321279 .

3. T. Jiang et al., Ultrafast fibe lasers mode-locked by twodimensional materials: review and prospect, Photonics Res. 8 (2020) 78, https: / / doi.org/10.1364/PRJ . 8 . 000078 .

4. W. Shi, Q. Fang, X. Zhu, R.A. Norwood, N. Peyghambarian, Fiber lasers and their applications, Appl. Opt. 53 (2014) 6554, https://doi.org/10.1364/AO.53.006554

5. B. Gao et al., Generation and categories of solitons in various mode-locked fibe lasers, Optik. 220 (2020) 165168, https: //doi.org/10.1016/j.ijleo.2020.165168

6. Y. Han, Y. Guo, B. Gao, C. Ma, R. Zhang, and H. Zhang, Generation, optimization, and application of ultrashort femtosec- ond pulse in mode-locked fibe lasers, Prog. Quantum. Electron, 71 (2020) 100264, https://doi.org/10.1016/ j.pquantelec.2020.100264.

7. W. Du, H. Li, Y. Lyu, C. Wei, and Y. Liu, Period doubling of dissipative-soliton-resonance pulses in passively mode-locked fibe lasers, Front. Phys. 7 (2020) 253, https: / / doi .org/ $10.3389 /$ fphy.2019.00253

8. Y. Wang et al., Breach and recurrence of dissipative soliton resonance during period-doubling evolution in a fibe laser, Phys. Rev. A 102 (2020) 013501, https: / / doi.org/10.1103/ PhysRevA.102.013501

9. F. O. Ilday, J. Buckley, and F. W. Wise, Period-doubling Route to Multiplepulsing in Femtosecond Fiber Lasers, in Nonlinear Guided Waves and Their Applications (Optical Society of America, Washington D.C., 2004), No. MD9, https: //doi.org/10.1364/NLGW.2004.MD9.

10. L. Larger, J.-P. Goedgebuer, and V. Udaltsov, Ikedabased nonlinear delayed dynamics for application to secure optical transmission systems using chaos, C. R. Phys. 5 (2004) 669, https://doi.org/10.1016/j.crhy. 2004.05 .003 
11. G. D. VanWiggeren and R. Roy, Optical communication with chaotic waveforms, Phys. Rev. Lett. 81 (1998) 3547, https : //doi.org/10.1103/PhysRevLett.81.3547.

12. A. K. Ghosh et al., Design of acousto-optic chaos based secure free-space optical communication links, in Proceedings of Free-Space Laser Communications IX (International Society of Optics and Photonics, California, 2009), Vol. 7464, https://doi.org/10.1117/12.826813

13. M. Iqbal, Z. Zheng, and T. Yu, Modeling of mode-locked lasers, Proceedings of the 7th WSEAS International Conference on Simulation, Modelling and Optimization. World Scientifi and Engineering Academy and Society (2007) 474.

14. S. Namiki, E.P. Ippen, H.A. Haus, and X.Y. Charles, Energy rate equations for mode-locked lasers, J. Opt. Soc. Am. B, 14 (1997) 2099, https://doi.org/10.1364/JOSAB . 14 . 002099

15. A. Haus, Hermann, J. G. Fujimoto, and P. I. Erich, Analytic theory of additive pulse and Kerr lens mode locking, IEEE J. Quantum Electron. 28 (1992) 2086, https : / / doi.org/ $10.1109 / 3.159519$

16. T.R. Schibli, E.R. Thoen, F.X. Kärtner, E.P. Ippen, Suppression of Q-switched mode locking and break-up into multiple pulses by inverse saturable absorption, Appl. Phys. B 70 (2000) S41, https://doi.org/10.1007/s003400000331.

17. M. A. Sharif, Modeling Mode-Locked Fiber Soliton Lasers based Optical Communication Networks, in Proceedings of th 5th Symposium on Adcances in Science and Technology (Civilica, Mashhad, 2011).

18. X. Liu, and B. Lee, A fast method for nonlinear Schrodinger equation, IEEE Photon. Technol. Lett. 15 (2003) 1549, https://doi.org/10.1109/LPT.2003.818679.

19. T. Chen, Z. Fang, Y. Hai-Qing, C. Zhan-Qing, and L. Tao, Three-Step Predictor-Corrector of Exponential Fitting Method for Nonlinear Schrödinger Equations, Commun. Theor. Phys. 44 (2005) 435, https://doi.org/10.1088/6102/ $44 / 3 / 435$

20. D. Kim, et al., Graphene-based saturable absorber and modelocked laser behaviors under gamma-ray radiation, Photonics Res. 7 (2019) 742, https://doi.org/10.1364/PRJ. 7.000742

21. G. Sobon, Mode-locking of fibe lasers using novel twodimensional nanomaterials: graphene and topological insulators, Photonics Res. 3 (2015) A56, https:// doi.org/10. $1364 / \mathrm{PRJ} .3 .000 \mathrm{~A} 56$

22. V. Mamidala, R. I. Woodward, Y. Yang, H.H. Liu, and K.K. Chow, Three-dimensional graphene based passively modelocked fibe laser, Opt. express 22 (2014) 31458, https: //doi.org/10.1364/OE.22.004539

23. H. Ahmad, F. D. Muhammad, M. Z. Zulkifli S. W. Harun, Graphene-based mode-locked spectrum-tunable fibe laser using Mach-Zehnder filte, IEEE Photonics J. 5 (2013) 1501709, https://doi.org/10.1109/JPHOT.2013. 2281609

24. Y. Hammadi et al., Graphene based soliton mode-locked erbium doped fibe laser for supercontinuum generation, Dig. J. Nanomater. Bios. 13 (2018) 777.

25. A. Ciattoni, and C. Rizza, Graphene-nonlinearity unleashing at lasing threshold in graphene-assisted cavities, Phys. Rev. A, 91 (2015) 053833, https://doi.org/10.1103/ PhysRevA.91.053833

26. N. A. Savostianova, and S. A. Mikhailov, Giant enhancement of the third harmonic in graphene integrated in a layered structure, Appl. Phys. Lett. 107 (2015) 181104, https://doi. org/10.1063/1.4935041. 